\title{
Evaluation of the efficacy of cisplatin-etoposide and the role of thoracic radiotherapy and prophylactic cranial irradiation in LCNEC
}

\author{
Arsela Prelaj ${ }^{1}$, Sara Elena Rebuzzi², Gabriella Del Bene ${ }^{1}$, \\ Julio Rodrigo Giròn Berrìos ${ }^{1}$, Alessandra Emiliani ${ }^{1}$, Lucilla De Filippis ${ }^{1}$, \\ Alessandra Anna Prete ${ }^{1}$, Silvia Pecorari ${ }^{1}$, Gaia Manna ${ }^{1}$, Carla Ferrara ${ }^{3}$, \\ Daniele Rossini ${ }^{4}$ and Flavia Longo ${ }^{1}$
}

Affiliations: 'Dept of Medical Oncology Unit A, Policlinico Umberto I, "Sapienza" University of Rome, Rome, Italy. ${ }^{2}$ Department of Medical Oncology, IRCCS San Martino IST, Genoa, Italy. ${ }^{3}$ Department of Public Health and Infectious Diseases, "Sapienza" University of Rome, Rome, Italy. ${ }^{4}$ Polo Oncologico, Azienda OspedalieroUniversitaria Pisana, Istituto Toscano Tumori, Pisa, Italy University of Pisa, Pisa, Italy.

Correspondence: Arsela Prelaj, Dept of Medical Oncology Unit A, Policlinico Umberto I, “Sapienza” University of Rome, Viale Regina Elena 324, 00161, Rome, Italy. E-mail: arsela201ahotmail.it

ABSTRACT In small-cell lung cancer (SCLC), the role of chemotherapy and radiotherapy is well established. Large-cell neuroendocrine carcinoma (LCNEC) shares several clinicopathological features with SCLC, but its optimal therapy is not defined. We evaluated clinical response and survival outcomes of advanced LCNEC treated in first-line therapy compared with SCLC.

72 patients with stage III-IV LCNEC $(\mathrm{n}=28)$ and extensive-stage SCLC (ES-SCLC) $(\mathrm{n}=44)$ received cisplatin-etoposide with/without thoracic radiotherapy (TRT) and prophylactic cranial irradiation (PCI).

Comparing LCNEC with SCLC, we observed similar response rates (64.2\% versus 59.1\%), disease control rates (82.1\% versus $88.6 \%$ ), progression-free survival (mPFS) (7.4 versus 6.1 months) and overall survival (mOS) (10.4 versus 10.9 months). TRT and PCI in both histologies showed a benefit in mOS (34 versus 7.8 months and 34 versus 8.6 months, both $\mathrm{p}=0.0001)$. LCNEC patients receiving TRT showed an improvement in mPFS and mOS (12.5 versus 5 months, $\mathrm{p}=0.02$ and 28.3 versus 5 months, $\mathrm{p}=0.004)$, similarly to ES-SCLC. PCI in LCNEC showed an increase in mPFS (20.5 versus 6.4 months, $\mathrm{p}=0.09$ ) and mOS (33.4 versus 8.6 months, $\mathrm{p}=0.05$ ), as in ES-SCLC.

Advanced LCNEC treated with SCLC first-line therapy has a similar clinical response and survival outcomes to ES-SCLC.

@ERSpublications

Cisplatin-etoposide is an efficient treatment for large-cell neuroendocrine carcinoma. RT and PCI improve survival. http://ow.ly/sBJo309HG8s

Cite this article as: Prelaj A, Rebuzzi SE, Del Bene G, et al. Evaluation of the efficacy of cisplatin-etoposide and the role of thoracic radiotherapy and prophylactic cranial irradiation in LCNEC. ERJ Open Res 2017; 3: 00128-2016 [https://doi.org/10.1183/23120541.00128-2016].

Received: Nov 162016 | Accepted after revision: Feb 122017

Conflict of interest: None declared.

Copyright $\odot$ ERS 2017. This article is open access and distributed under the terms of the Creative Commons Attribution Non-Commercial Licence 4.0. 


\section{Introduction}

Neuroendocrine tumours (NETs) are a heterogeneous group of neoplasms that arise from cells of the neuroendocrine system. Pulmonary NETs represent approximately $20 \%$ of all lung cancers and show a spectrum of histology, clinical profiles and biological behaviours that range from low-grade and relatively indolent carcinoids (typical and atypical carcinoids) to aggressive high-grade neuroendocrine carcinomas (HGNECs), including large-cell neuroendocrine carcinomas (LCNECs) and small-cell lung cancer (SCLC) $[1,2]$.

SCLC is the most frequent lung NET, representing approximately $15 \%$ of lung cancers, while LCNEC accounts for about 3\% of all primary lung cancers [3]. LCNEC was first included into the World Health Organization (WHO) classification of lung cancer in 2004 as a variant of large-cell carcinoma [4]. In the 2015 WHO classification, this tumour is no longer classified under large-cell carcinomas, but in a group of neuroendocrine neoplasms including SCLC, typical carcinoids and atypical carcinoids [5].

LCNEC and SCLC shares several clinico-pathological features, including aggressive clinical behaviour, poor prognosis, predominance in males, strong link to smoking and some histological features [6, 7]. In addition, LCNEC and SCLC have common genetic alterations and neuroendocrine gene expression $[8,9]$.

Because of the rarity and complex histopathological characteristics of LCNEC, its optimal therapeutic management has not yet been defined and it remains uncertain whether LCNEC patients should be treated according to non-small cell lung cancer (NSCLC)-based or SCLC-based regimes [6]. The international guidelines $[10,11]$ recommend that the treatment management of LCNEC should follow the NSCLC algorithm. However, they report that chemotherapy regimens commonly used for SCLC may represent the most reasonable treatment option on the basis of several clinical trials [7, 12-14].

Some trials have reported that LCNEC responds to cisplatin-based chemotherapeutic regimens similar to those used for SCLC [13-15], while others have separated chemotherapy regimens into NSCLC-type and SCLC-type, demonstrating a difference in survival, and favouring SCLC-type chemotherapy [7, 12]. These different responses may be based on the overexpression of different driver genes (topoisomerase II, somatostatin, ERCC1) compared with adenocarcinoma, which leads to a more favourable response to SCLC treatment with etoposide and octreotide, and resistance to NSCLC regimens [16].

Regarding the role of radiotherapy in HGNEC, in limited-stage SCLC (LS-SCLC), thoracic radiotherapy (TRT) concurrent with chemotherapy and prophylactic cranial irradiation (PCI) is recommended [10]. Consolidative TRT is beneficial for selected patients with extensive-stage SCLC (ES-SCLC) who respond to chemotherapy, in terms of the reduction of symptoms, control of chest recurrences and improvement in long-term survival [10, 17-19]. PCI in patients with ES-SCLC reduces the incidence of brain metastasis, but the impact on overall survival is controversial $[20,21]$. The role of TRT and PCI in local or advanced LCNEC remains undefined $[6,22,23]$.

The aim of this retrospective study is to report our experience in the treatment of HGNEC and, in particular, to evaluate the effectiveness of the combination of cisplatin and etoposide as first-line therapy in LCNEC, with or without TRT and PCI, compared to SCLC.

\section{Materials and methods}

Study population

This retrospective study was approved by the Institutional Review Board of Policlinico Umberto I, Sapienza University of Rome. From the database of Dept of Oncology Unit A, we performed a retrospective analysis of adult patients affected by ES-SCLC and advanced (stage IIIA-IIIB) or metastatic (stage IV) LCNEC of the lung, treated with a combination of cisplatin and etoposide as first-line therapy, with or without sequential TRT and PCI. The extensive stage of SCLC included distant metastases (stage IV) or T3-T4 (stage III) due to multiple lung nodules that are too extensive or do not fit in a tolerable radiation field. For all patients, diagnosis was established histologically using WHO criteria, and radiologically by total body computed tomography (TB CT) scan.

Clinical data included patients' characteristics (sex, age, performance status, smoking history), tumour characteristics (histotype, clinical stage at diagnosis and pre-treatment, site of disease, brain metastasis) and treatment information (surgery at diagnosis, type of surgery, median cycles, TRT, PCI, next lines of chemotherapy).

\section{Treatment plan}

All patients included in the analysis underwent first-line therapy with a combination of cisplatin $75 \mathrm{mg} \cdot \mathrm{m}^{-2}$ at day 1 and etoposide $100 \mathrm{mg} \cdot \mathrm{m}^{-2}$ on days $1-3$ every 3 weeks, with or without sequential TRT and PCI. TRT was delivered to a dose of $60 \mathrm{~Gy}$ in 10 fractions after six cycles of chemotherapy, and PCI was administered with a total dose of $25 \mathrm{~Gy}$ in 10 fractions. 


\section{Response evaluation}

All patients were followed clinically by a multidisciplinary team. Radiological tumour assessment was performed by TB CT at baseline and after the first three cycles of chemotherapy, and then every two cycles or whenever progression of disease was clinically suspected. Tumour response was assessed according to the Response Evaluation Criteria in Solid Tumors (RECIST), version 1.1 [24] as complete $(\mathrm{CR})$ and partial (PR) response, stable (SD) and progression (PD) of disease. Response rate (RR) was defined as the sum of CR and PR, while disease control rate (DCR) was defined as the sum of CR, PR and $\mathrm{SD}$. Diagnosis of recurrence was determined by TB CT scan, or cerebral magnetic resonance imaging (MRI) in the case of suspected brain metastasis.

\section{Statistical analysis}

Survival analysis was conducted on the efficacy of first-line chemotherapy with the combination of cisplatin and etoposide in HGNEC of the lung, in particular LCNEC compared to SCLC, in terms of median progression-free survival (mPFS) and overall survival (mOS), 6 months and 12 months PFS (PFS-6, PFS-1y) and OS (OS-6, OS-1y) from the start of chemotherapy.

Survival analysis was also conducted on the efficacy of TRT and PCI both in SCLC and LCNEC and different survival based on disease localisation (thoracic or extrathoracic).

PFS was measured from the first chemotherapy administration to diagnosis of PD (evidenced by TB CT scan) or to death, or to last follow-up assessment. OS was measured from the first chemotherapy administration to death or last follow-up.

Median PFS and OS were estimated with their 95\% confidence interval (CI). Survival curves of PFS and OS were generated using the Kaplan-Meier method [25]. Differences in PFS and OS were evaluated using the log-rank test (Mantel-Cox) for statistical significance, which was defined at the $\mathrm{p}<0.05$ level [25].

\section{Results}

\section{Patient characteristics}

Between January 2004 and February 2016, a total of 72 patients with advanced or metastatic HGNEC, receiving first-line cisplatin-etoposide chemotherapy with or without TRT and PCI, were included in the analysis. Patient, tumour and treatment characteristics are summarised in table 1.

Most patients were male (64\%) and had a smoking history (89\%). The median age was 64 years (range 40-80) and the median PS was 0 . All patients had a histological diagnosis of HGNEC, 44 patients were affected by ES-SCLC $(\mathrm{n}=44,61 \%)$ and 28 patients were affected by advanced (stage IIIA-IIIB) or metastatic (stage IV) LCNEC of the lung $(n=28,9 \%)$. The disease localisation was intrathoracic in $51.4 \%$ of patients.

All patients received combination chemotherapy with cisplatin and etoposide as first-line treatment, while $36.1 \%$ of patients were treated with sequential TRT and $29.2 \%$ of patients with PCI. All patients received at least three administrations of cisplatin-etoposide. The median number of cycles administered was six (range 3-8). After progressive disease, $45.8 \%$ of patients underwent further lines of chemotherapy: 33 patients (45.8\%) underwent a second-line chemotherapy and 9 patients (12.5\%) up to a third-line therapy.

\section{Treatment response (activity)}

All patients included in the study were assessable for response analysis (table 2). Among the 28 patients with LCNEC, 2 patients had a CR (7.1\%), 16 patients achieved PR (57.1\%) and 5 patients SD (17.9\%) with a RR of $64.2 \%$ and a DCR of $82.1 \%$. Five patients (17.9\%) experienced PD.

Among the 44 patients with SCLC, 1 patient had a CR (2.3\%), 25 patients achieved PR (56.8\%) and 13 patients SD (29.5\%), with a RR of $59.1 \%$ and a DCR of $88.6 \%$. Five patients (11.4\%) experienced PD.

\section{Efficacy evaluation}

From statistical analysis, LCNEC presented similar values of DCR, mPFS and mOS as SCLC (figure 1). The mPFS was 7.4 months (95\% CI 4.7-10.1) for LCNEC and 6.1 months (95\% CI 4.9-7.3) for SCLC, with a PFS-6 of 53.6 and 52.3 months, respectively. The mOS was 10.4 months (95\% CI 7.3-13.5) for LCNEC and 10.9 months (95\% CI 6-15.8) for SCLC. The results are summarised in table 2.

\section{Activity according to sequential thoracic radiotherapy}

The subgroup analysis of 26 HGNEC patients (16 SCLC and 10 LCNEC) that completed chemotherapy (indicated by "CT" in table 3), with sequential TRT after six cycles of chemotherapy, showed longer survival (figure 1), with a mOS of 34 months (95\% CI 25.7-42.3 months) versus 7.8 months (95\% CI 5.3-10.3 months) in patients that did not received TRT (reaching statistical significance, $\mathrm{p}=0.0001$ ). Statistical significance is 


\begin{tabular}{|c|c|c|}
\hline Characteristics & LCNEC & SCLC \\
\hline Subjects & $28(39 \%)$ & $44(61 \%)$ \\
\hline \multicolumn{3}{|l|}{ Sex } \\
\hline Male & $18(64.3 \%)$ & $28(63.6 \%)$ \\
\hline Female & 10 (35.7\%) & $16(36.4 \%)$ \\
\hline Age years & $65(40-78)$ & $64(46-80)$ \\
\hline \multicolumn{3}{|l|}{ ECOG performance status } \\
\hline 0 & $15(53.6 \%)$ & $34(54.5 \%)$ \\
\hline 1 & $9(32.1 \%)$ & $12(27.3 \%)$ \\
\hline 2 & $4(14.3 \%)$ & $8(18.2 \%)$ \\
\hline \multicolumn{3}{|l|}{ Smoking history } \\
\hline Never smoker ${ }^{\#}$ & $4(14.3 \%)$ & $4(9.1 \%)$ \\
\hline Ever smoker & $24(85.7 \%)$ & $40(90.9 \%)$ \\
\hline \multicolumn{3}{|l|}{ Clinical stage at first-line therapy } \\
\hline III & 10 (35.7\%) & \\
\hline IV & $18(64.3 \%)$ & \\
\hline \multicolumn{3}{|l|}{ Clinical stage at diagnosis } \\
\hline$|-| \mid$ & $6(21.4 \%)$ & $2(4.5 \%)$ \\
\hline IIIA/IIIB & $7(25 \%)$ & $14(31.9 \%)$ \\
\hline IV & $15(53.6 \%)$ & $28(63.6 \%)$ \\
\hline \multicolumn{3}{|l|}{ Site of disease } \\
\hline Intrathoracic & $13(46.4 \%)$ & $24(54.5 \%)$ \\
\hline Extrathoracic & $15(53.6 \%)$ & $20(45.5 \%)$ \\
\hline \multicolumn{3}{|l|}{ Brain metastasis at diagnosis } \\
\hline Yes & $2(7.1 \%)$ & $4(90.9 \%)$ \\
\hline No & $26(92.9 \%)$ & $40(9.1 \%)$ \\
\hline \multicolumn{3}{|l|}{ Surgery at diagnosis } \\
\hline Yes & $6(21.4 \%)$ & $2(4.5 \%)$ \\
\hline No & $22(78.6 \%)$ & $42(95.5 \%)$ \\
\hline \multicolumn{3}{|l|}{ Type of surgery } \\
\hline Atypical resection & $1(3.6 \%)$ & $0(0 \%)$ \\
\hline Lobectomy & $3(10.7 \%)$ & $2(4.5 \%)$ \\
\hline Lobectomy+lymphadenectomy & $2(7.1 \%)$ & $0(0 \%)$ \\
\hline \multicolumn{3}{|l|}{ Chemotherapy treatment } \\
\hline Cycles received & $6(3-8)$ & $6(3-8)$ \\
\hline \multicolumn{3}{|l|}{ Consolidative TRT } \\
\hline Yes & $10(35.7 \%)$ & $16(36.4 \%)$ \\
\hline No & $18(64.3 \%)$ & $28(63.6 \%)$ \\
\hline \multicolumn{3}{|l|}{$\mathrm{PCl}$} \\
\hline Yes & $4(14.3 \%)$ & $17(38.6 \%)$ \\
\hline No & $24(85.7 \%)$ & $27(61.4 \%)$ \\
\hline \multicolumn{3}{|l|}{ Successive lines of chemotherapy } \\
\hline None & $14(50 \%)$ & $16(36.4 \%)$ \\
\hline$\|$ & $12(42.9 \%)$ & $21(47.7 \%)$ \\
\hline III & $2(7.1 \%)$ & $7(15.9 \%)$ \\
\hline
\end{tabular}

Data are presented as $\mathrm{n}(\%)$ or median (range). LCNEC: large cell neuroendocrine carcinoma; SCLC: small cell lung cancer; TRT: thoracic radiotherapy; $\mathrm{PCl}$ : prophylactic cranial irradiation. ${ }^{\#}:<100$ cigarettes in lifetime.

reached for mPFS and mOS both for LCNEC and SCLC (table 3). Among LCNEC patients, those treated with chemotherapy and sequential TRT experienced higher mPFS (12.5 versus 5 months, $\mathrm{p}=0.02)$ and mOS (28.3 versus $5, \mathrm{p}=0.004)$. Also, SCLC patients treated with chemotherapy and sequential TRT showed higher mPFS (9.5 versus 4.6 months, $\mathrm{p}=0.0001$ ) and $\operatorname{mOS}(34.3$ versus $8.1, \mathrm{p}=0.0001$ ) (figure 2 and table 3 ).

\section{Activity according to $\mathrm{PCI}$}

The subgroup analysis of 21 patients (17 SCLC and 4 LCNEC) that were treated with PCI after first-line chemotherapy showed longer survival (figure 1), with a mOS of 34 months (95\% CI 32.4-35.6 months) versus 8.6 months (95\% CI 6.2-11 months) in patients that did not received PCI (reaching statistical significance, $\mathrm{p}=0.001$ ). Statistical significance in terms of mPFS and mOS is reached only for SCLC (table 3 ). 


\begin{tabular}{|c|c|c|}
\hline Objective responses & LCNEC & SCLC \\
\hline Complete response & $2(7.1 \%)$ & $1(2.3 \%)$ \\
\hline Partial response & $16(57.1 \%)$ & $25(56.8 \%)$ \\
\hline Stable disease & $5(17.9 \%)$ & $13(29.5 \%)$ \\
\hline Progressive disease & $5(17.9 \%)$ & $5(11.4 \%)$ \\
\hline Response rate & $18(64.2 \%)$ & $26(59.1 \%)$ \\
\hline Disease control & $23(82.1 \%)$ & $39(88.6 \%)$ \\
\hline \multicolumn{3}{|l|}{ Survival data } \\
\hline PFS months & $7.4(1-92.4)$ & $6.1(1-130.2)$ \\
\hline 6-month PFS & $53.6 \%$ & $52.3 \%$ \\
\hline 12-months PFS & $25 \%$ & $15.9 \%$ \\
\hline OS months & $10.4(1-84.4)$ & $10.9(1.6-130.2)$ \\
\hline 6-month OS & $64.3 \%$ & $75 \%$ \\
\hline 12-months OS & $35.7 \%$ & $38.6 \%$ \\
\hline
\end{tabular}

Among LCNEC patients, those treated with PCI experienced higher mPFS (20.5 versus 6.4 months) and mOS (33.4 versus 8.6 months) without reaching statistical significance ( $\mathrm{p}=0.09$ and $\mathrm{p}=0.05$ respectively). SCLC patients treated with PCI showed higher mPFS (8.6 versus 4.6 months) and mOS (34 versus 8.4 months), reaching statistical significance (both $\mathrm{p}=0.0001$ ) (figure 3 and table 3 ).

\section{Activity according to stage in SCLC}

A subgroup analysis was performed according to stage in SCLC patients. Stage III SCLC patients experienced longer survival, with a mOS of 18.4 versus 9.2 months and a mPFS of 9.2 versus 5.4 months compared to stage IV SCLC patients, reaching statistical significance ( $\mathrm{p}=0.04$ and $\mathrm{p}=0.02$, respectively).

A survival analysis of the use of TRT in SCLC according to stage reported statistically significant differences both in stage III and stage IV SCLC. Stage III SCLC treated with TRT had a survival benefit in mOS of 23.4 months (34.3 versus 10.9 months; $\mathrm{p}=0.004$ ) and in mPFS of 5.3 months (9.5 versus 4.2 months; $\mathrm{p}=0.009)$. Stage IV SCLC treated with TRT had a survival benefit in mOS of 6.9 months (14.4 versus 7.5 months; $\mathrm{p}=0.03$ ) and in $\mathrm{mPFS}$ of 3.2 months ( 7.8 versus 4.6 months; $\mathrm{p}=0.008$ ).

Survival analysis of the use of PCI in SCLC according to stage reported statistically significant differences both in stage III and stage IV SCLC, except for mOS in stage IV SCLC, which had a trend towards statistical significance. Stage III SCLC treated with PCI had a survival benefit in mOS of 23.4 months (34.3 versus 10.9 months; $\mathrm{p}=0.001)$ and in $\mathrm{mPFS}$ of 8.4 months (12.6 versus 4.2 months; $\mathrm{p}=0.01)$. Stage IV SCLC treated with PCI had a survival benefit in mOS of 6.9 months (14.4 versus 7.5 months; $\mathrm{p}=0.09)$ and in mPFS of 3.2 months (7.8 versus 4.6 months; $\mathrm{p}=0.004$ ).

\section{Activity according to stage in LCNEC}

A subgroup analysis was performed according to stage in LCNEC patients. Stage III LCNEC experienced longer survival, with a mOS of 28.3 versus 4.9 months and a mPFS of 12.5 versus 4.5 compared to stage IV LCNEC, reaching statistical significance $(\mathrm{p}=0.001$ and $\mathrm{p}=0.004$, respectively). The survival analysis of the use of TRT and PCI in LCNEC according to stage did not report statistically significant differences, probably due to the low number of patients, except for a benefit in PFS with the use of TRT (14 versus 9.6 months; $\mathrm{p}=0.04)$ and PCI (20.5 versus 12 months; $\mathrm{p}=0.03)$ in stage III LCNEC patients. In mOS, a trend towards statistical significance was observed in stage III LCNEC treated with TRT (33.4 versus 10.4 months, $\mathrm{p}=0.06$ ).

\section{Activity according to disease localisation}

Advanced or metastatic LCNEC patients with intrathoracic disease showed higher mPFS (10.8 versus 5.1 months) and mOS (13.3 versus 8 months) compared to those with extrathoracic disease, reaching statistical significance $(\mathrm{p}=0.02$ and $\mathrm{p}=0.04$, respectively). Also, ES-SCLC patients with intrathoracic disease had higher mPFS (7.8 versus 4.9 months) and mOS (15.4 versus 8.4 months), but without reaching statistical significance (both $\mathrm{p}=0.06$ ). 

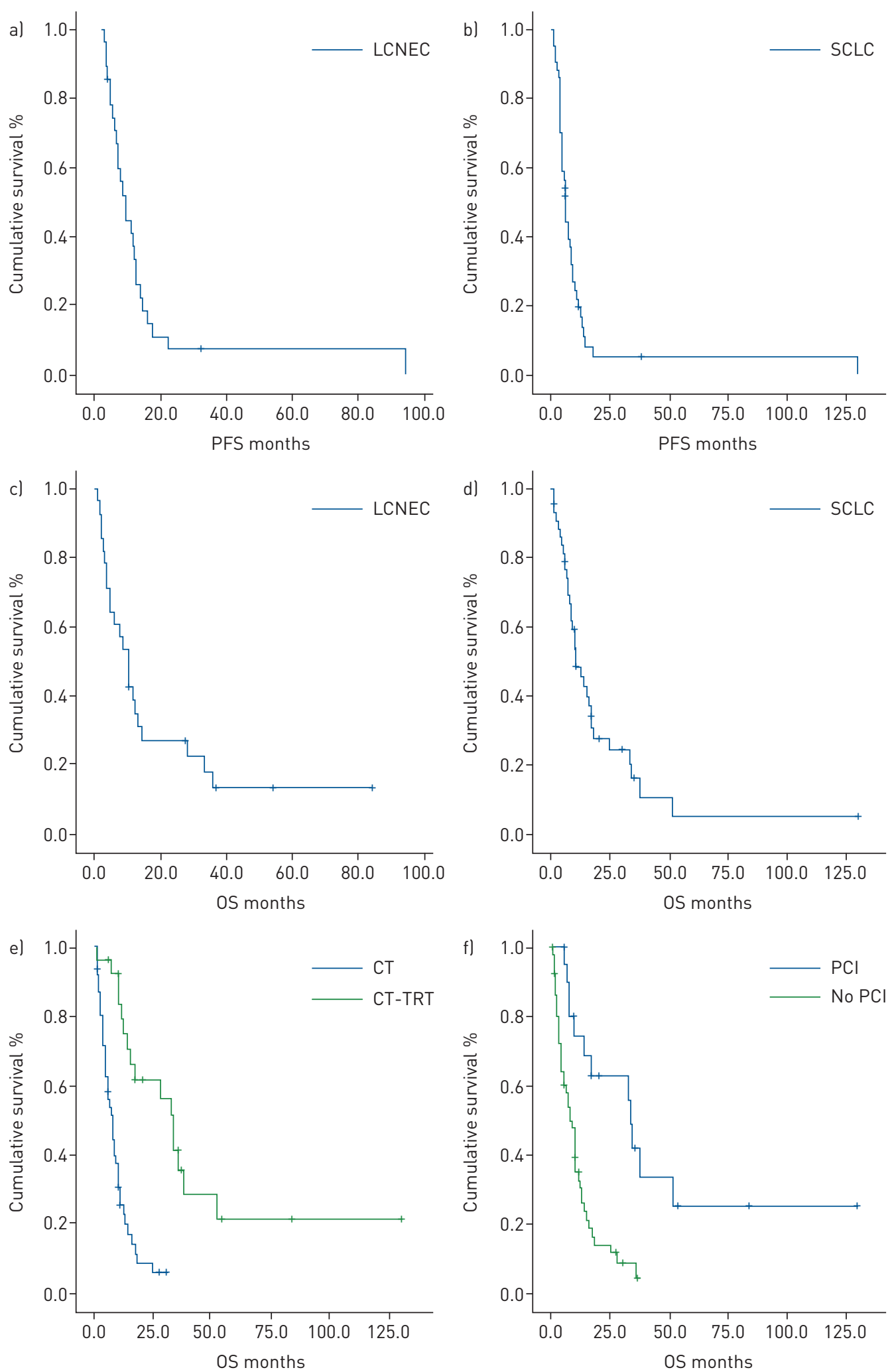

FIGURE 1 a-d) Comparison of Kaplan-Meier curves of a) progression-free survival (PFS) and c) overall survival (OS) in large-cell neuroendocrine carcinoma (LCNEC) patients undergoing first-line therapy with cisplatin-etoposide and Kaplan-Meier curves of b) PFS and d) OS in small-cell lung cancer (SCLC) patients undergoing the same therapy. e, f) Kaplan-Meier curves for OS according to the use of e) thoracic radiotherapy (TRT) and f) prophylactic cranial irradiation (PCI) following first-line chemotherapy (CT) in high-grade neuroendocrine carcinoma patients. 


\begin{tabular}{|c|c|c|c|c|}
\hline & mPFS months $(95 \% \mathrm{CI})$ & p-value & mOS months $(95 \% \mathrm{CI})$ & p-value \\
\hline \multicolumn{5}{|l|}{ SCLC } \\
\hline First-line treatment & & 0.0001 & & 0.0001 \\
\hline CT-TRT & $9.5(4.6-14.5)$ & & $34.3(10.7-57.9)$ & \\
\hline CT & $4.6(4.1-5.1)$ & & $8.1(6.6-9.6)$ & \\
\hline $\mathrm{PCl}$ & & 0.0001 & & 0.001 \\
\hline Yes & $8.6(6.2-11)$ & & $34(10.6-57.4)$ & \\
\hline No & $4.6(4.1-5.1)$ & & $8.4(4.9-11.9)$ & \\
\hline Stage & & 0.02 & & \\
\hline III & $9.2(7.7-10.7)$ & & $18.4(14.9-21.9)$ & \\
\hline IV & $5.4(3.8-7)$ & & $9.2(6-12)$ & \\
\hline Site of disease & & 0.06 & & 0.06 \\
\hline Thoracic & $7.8(4.3-11.3)$ & & $15.4(10.7-20.1)$ & \\
\hline Extrathoracic & $4.9(2.3-7.5)$ & & $8.4(6.1-10.7$ & \\
\hline \multicolumn{5}{|l|}{ LCNEC } \\
\hline First-line treatment & & 0.02 & & 0.04 \\
\hline CT-TRT & $12.5(11.1-13.9)$ & & $28.3(0-60.7)$ & \\
\hline CT & $5(3.8-6.2)$ & & $5(2.7-7.3)$ & \\
\hline $\mathrm{PCl}$ & & 0.09 & & 0.05 \\
\hline Yes & $20.5(0-46.7)$ & & $33.4(8-53.4)$ & \\
\hline No & $6.4(3.4-9.4)$ & & $8.6(3.4-13.8)$ & \\
\hline Stage & & 0.004 & & \\
\hline III & $12.5(11-14)$ & & $28.3(0-59.4)$ & \\
\hline IV & $4.5(2.6-6.4)$ & & $4.9(2.2-7.6)$ & \\
\hline Site of disease & & 0.02 & & 0.04 \\
\hline Thoracic & $10.8(8.8-12.8)$ & & $13.3(0-33)$ & \\
\hline Extrathoracic & $5.1(2.7-7.5)$ & & $8(3.5-12.5)$ & \\
\hline
\end{tabular}

\section{Discussion}

LCNEC of the lung is a rare neuroendocrine pulmonary tumour that shares several clinicopathological and genetic features with SCLC [6-9]. Because of its rarity and complex histopathology, there is no consensus on the standard treatment for advanced LCNEC, especially regarding the use of NSCLC-based or SCLC-based chemotherapy [6]. Although the international guidelines [10, 11] recommend the NSCLC algorithm, several clinical trials have reported interesting results with SCLC-based chemotherapy $[7,12-14]$. Positive results were observed with SCLC regimens in an adjuvant setting encouraged to use this strategy in unresectable LCNEC [23, 26-28].

In 2005, Rossi et al. [12] analysed 83 pulmonary LCNEC, exploring clinical and therapeutic histories, and observed that cisplatinum-etoposide chemotherapy, a typical SCLC regimen, reported higher RR and longer survival than traditional NSCLC regimens, both in adjuvant and metastatic settings. Many studies then confirmed these results, comparing NSCLC and SCLC chemotherapy, such as the trial by SuN et al. [7]. Other trials have reported that RR and survival outcomes for LCNEC with cisplatin-based chemotherapy are comparable to SCLC, both with cisplatin-etoposide [13-15, 22] and cisplatin-irinotecan [13, 15, 29-32].

We conducted a retrospective analysis on ES-SCLC and advanced (stage IIIA-IIIB) or metastatic (stage IV) LCNEC of the lung treated with cisplatin-etoposide as first-line therapy with or without sequential TRT and PCI. Comparing the LCNEC group with the SCLC group, we generally observed similar values of RR (64.2\% versus $59.1 \%)$, DCR (82.1\% versus $88.6 \%$ ), mPFS (7.4 versus 6.1 months) and mOS (10.4 versus 10.9 months) (table 2 and figure 1), confirming the similar clinical behaviour and outcomes for the two histologies, as reported in the literature. The survival outcomes for both LCNEC and SCLC are longer for stage III than for stage IV, with statistical significance.

Regarding the role of radiotherapy, in LS-SCLC the addition of concurrent TRT with chemotherapy improves long-term survival and is currently the standard therapy [10, 33-35]. In ES-SCLC, consolidative TRT has been found to be beneficial for selected patients who respond to chemotherapy, in terms of the reduction of symptoms, control of chest recurrences and improvement of long-term survival, on the basis of several retrospective and prospective studies $[10,17-19,36]$. 

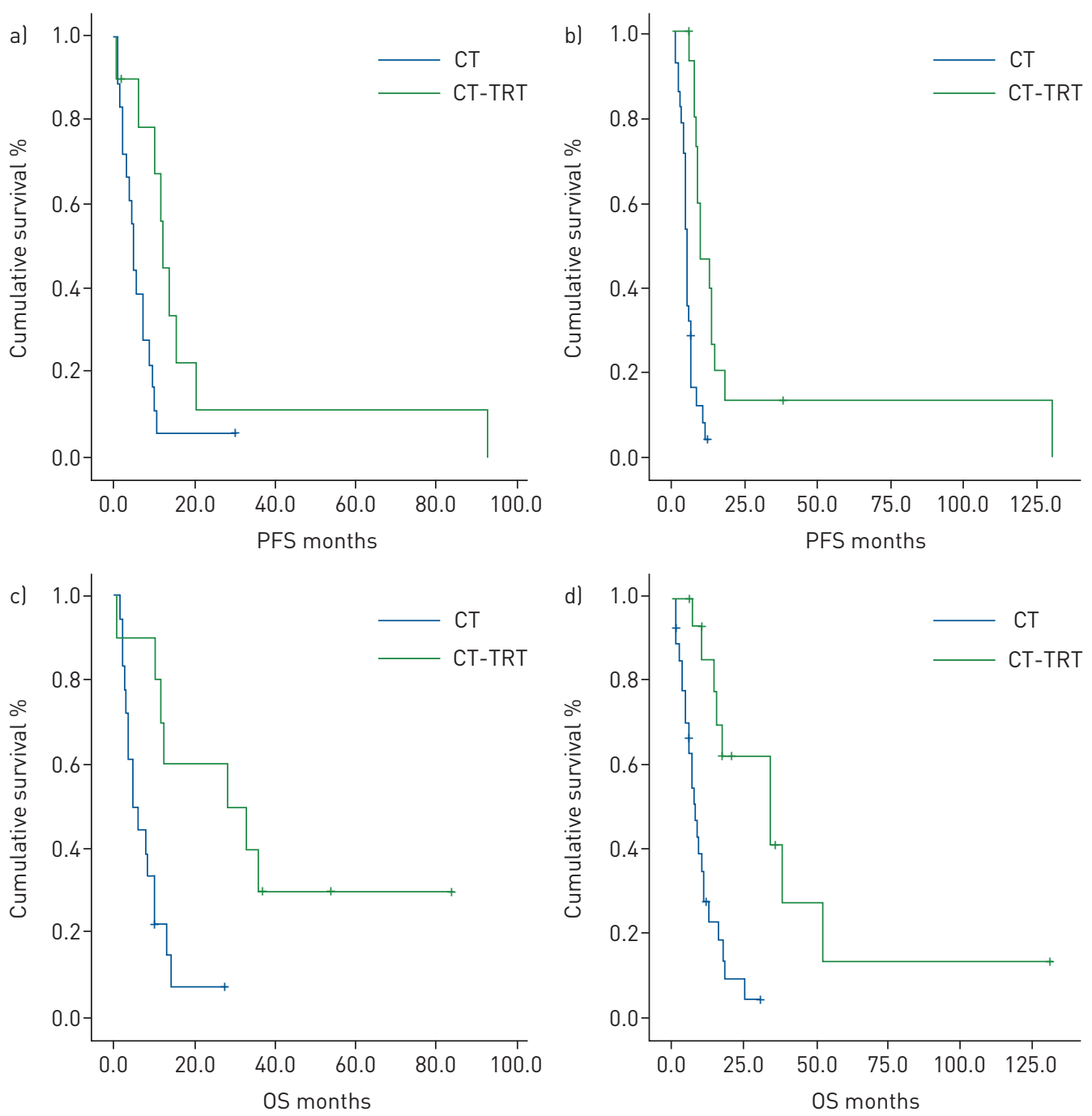

FIGURE 2 Kaplan-Meier curves for a, b) progression-free survival (PFS) and c, d) overall survival (OS) in a, c) large-cell neuroendocrine carcinoma patients and b, d) small-cell lung cancer patients according to the use of thoracic radiotherapy (TRT). CT: chemotherapy.

In 1999, JEREMIC et al. performed a randomised study on ES-SCLC patients with a CR outside the thorax and at least a PR inside the thorax after three cycles of cisplatin-etoposide [17]. The patients were randomised between chemotherapy or TRT plus chemotherapy. The authors observed an improvement in 5 year OS in the group receiving TRT. Similar results were reported by YEE et al. in 2012 [18]. In 2015, SLOTMAN et al. [19] performed a phase III randomised trial on consolidative TRT in ES-SCLC patients (CREST trial) who responded to four to six cycles of cisplatin-etoposide followed by PCI. They showed a longer mPFS in the TRT group compared to the control group ( 4 versus 3 months, $\mathrm{p}=0.001$ ) and a mOS of 8 months in both groups, but with a higher OS rate at 1 year and at 2 years in the TRT group. This study confirmed that TRT in addition to PCI should be considered for all patients with ES-SCLC who respond to chemotherapy. Other prospective and retrospective studies reported positive results with TRT in ES-SCLC in terms of survival and RR [37-39].

The role of TRT in local or advanced LCNEC remains undefined $[6,22,23]$ due to the lack of prospective studies. Only Metro et al. [22] have conducted a retrospective study on the therapeutic management of advanced LCNEC in terms of the efficacy of cisplatin-etoposide chemotherapy with or without TRT and/ or PCI compared to SCLC. The TRT was administered as a radical and palliative radiotherapy and concomitantly and sequentially to chemotherapy. They reported lower RR and DCR, mPFS (5.6 versus 8.9 months) and mOS (10.4 versus 17.6 months) for LCNEC compared to SCLC.

In our retrospective study we analysed the role of consolidative TRT in advanced LCNEC compared to ES-SCLC. Our general results on the use of TRT in HGNEC (LCNEC+SCLC) showed a statistically significant 

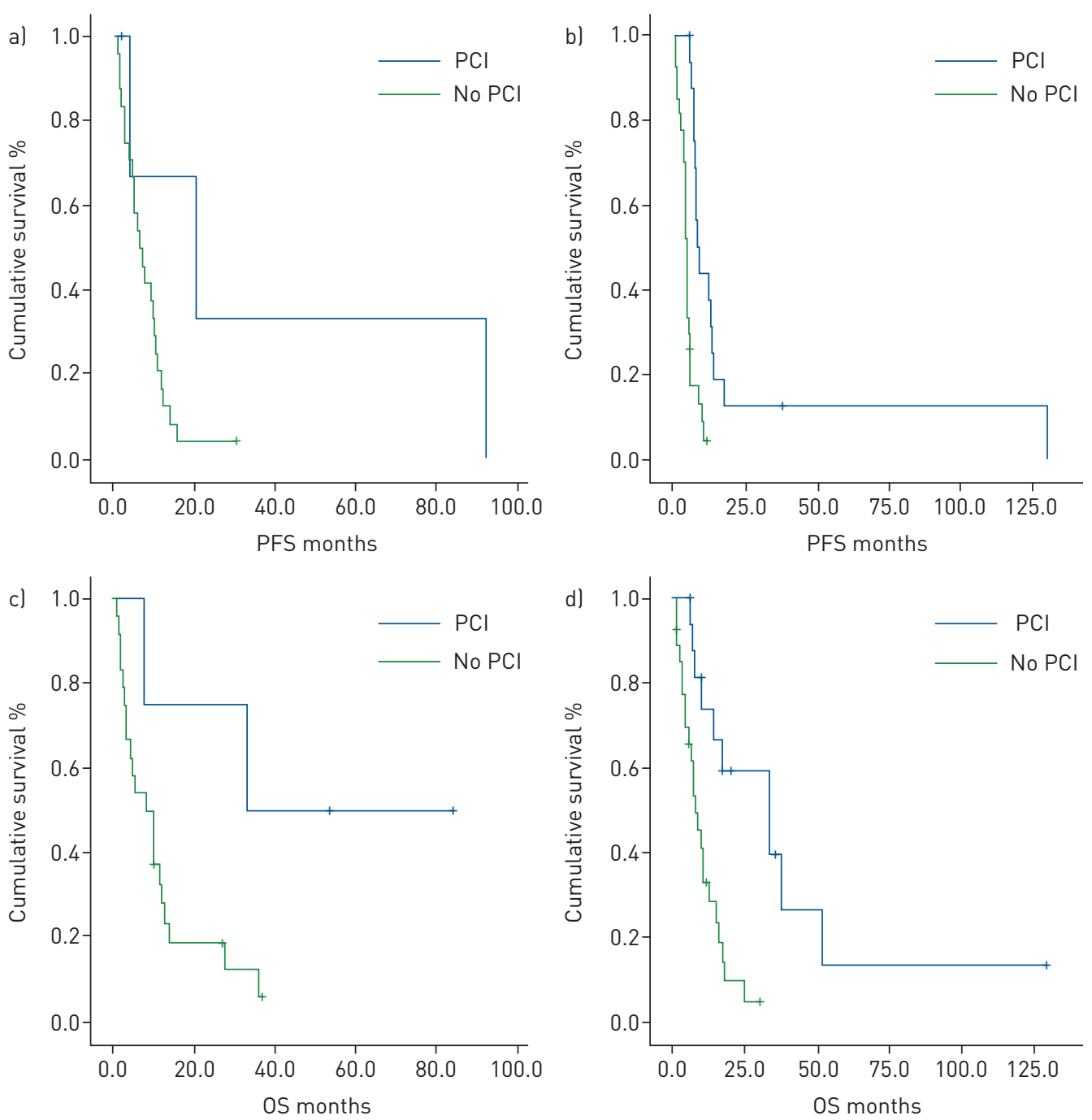

FIGURE 3 Kaplan-Meier curves for a, b) progression-free survival (PFS) and c, d) overall survival (OS) in a, c) large-cell neuroendocrine carcinoma patients and b, d) small-cell lung cancer patients according to the use of prophylactic cranial irradiation (PCI).

benefit in terms of OS (34 versus 7.8 months, $\mathrm{p}=0.0001$ ) (figure 1). Regarding SCLC, we confirmed the beneficial role of consolidative TRT in ES-SCLC in terms of mPFS and mOS, reaching statistical significance (9.5 versus 4.6 months, $\mathrm{p}=0.0001 ; 34.3$ versus 8.1 months, $\mathrm{p}=0.0001$, respectively). Most importantly, we reported higher survival results in advanced LCNEC patients receiving consolidative TRT, reaching statistical significance both in terms of mPFS and mOS ( 12.5 versus 5 months, $\mathrm{p}=0.02 ; 28.3$ versus 5 months, $\mathrm{p}=0.004$, respectively) (table 3 and figure 2), and more evident for stage III than for stage IV LCNEC.

The role of PCI in LS-SCLC was established in 1999 with a meta-analysis of randomised trials, which demonstrated that PCI decreased brain metastases and increased OS in patients with LS-SCLC who have a good response to chemotherapy [40]. In 2007, SLotman et al. [20] conducted a phase III trial on patients with ES-SCLC who had responded to first-line chemotherapy and were randomly assigned to receive either PCI or no PCI. They demonstrated that PCI reduced the incidence of brain metastases (cumulative risk at 1 year of $40.4 \%$ versus $14.6 \%, \mathrm{p}<0.001)$ and improved OS at 1 year $(27.1 \%$ versus $13.3 \%, \mathrm{p}=0.003)$ compared with the control group. Preliminary results of a Japanese phase III trial [21] reported a reduced risk of developing brain metastases at 1 year $(32.2 \%$ versus $58 \%, \mathrm{p}<0.001)$, but did not show a benefit in terms of survival. Despite these contrasting results, PCI is currently recommended in ES-SCLC patients who respond to first-line chemotherapy $[10,41]$.

The role of PCI in local or advanced LCNEC is still undefined $[6,22,23]$, even though advanced LCNEC are associated with a high rate of brain recurrence and the use of PCI could provide some benefit in terms of control of brain relapses and OS, as in ES-SCLC. 
In our retrospective analysis we studied the role of PCI in advanced LCNEC compared to ES-SCLC. Our general results on the use of PCI in HGNEC (LCNEC+SCLC) showed a statistically significant benefit in terms of overall survival (34 versus 8.6 months, $\mathrm{p}=0.0001$ ) (figure 1). Regarding SCLC, we confirmed the beneficial role of PCI in ES-SCLC in terms of mPFS and mOS, reaching statistical significance (8.6 versus 4.6 months, $\mathrm{p}=0.0001 ; 34$ versus 8.4 months, $\mathrm{p}=0.0001$, respectively), which was more evident for stage III than for stage IV SCLC. We reported higher survival results in advanced LCNEC patients receiving PCI, with just a trend towards statistical significance, both in terms of mPFS and mOS, probably due to the unbalance between the two treatment groups (20.5 versus 6.4 months, $\mathrm{p}=0.09 ; 33.4$ versus 8.6 months, $\mathrm{p}=0.05$, respectively) (table 3 and figure 3 ). The survival benefit with PCI is more evident for stage III than for stage IV LCNEC.

The main limitations of this study are its retrospective nature, the lack of selection of the population analysed, the relatively low sample size and the unbalanced numbers of patients in the different groups in terms of pathology and treatment (LCNEC/SCLC, TRT/No TRT, PCI/No PCI).

Our results, despite these limitations, showed clinical responses and survival rates in LCNEC and SCLC that are similar to those seen in the literature, confirming the similar clinico-pathological behaviour of the two histologies. Moreover, our study reported statistically significative survival results with the use of TRT and PCI, both in LCNEC and SCLC. No revisions of histologies were needed, so there were no misdiagnoses between LCNEC and SCLC.

To the best of our knowledge, our study is the first to evaluate the role of cisplatin-etoposide chemotherapy with and without TRT and PCI in advanced LCNEC, and to compare the results with those for SCLC. Our study has shown not only that LCNEC has a similar clinical behaviour to SCLC, but also that TRT in advanced LCNEC gives a statistically significant survival benefit, similar to the situation with LS- and ES-SCLC. Furthermore, PCI in advanced LCNEC gave a survival benefit similar to that in SCLC, but without reaching statistical significance, probably due to the unbalanced size of the two groups of treatment and the low number of LCNEC patients analysed.

Future phase III studies are needed to establish which chemotherapy regimen - NSCLC-based chemotherapy or SCLC-based chemotherapy - is the best option for the treatment of LCNEC.

We also believe that our results form the basis for a reasonable rationale for a larger prospective study to determine the role of TRT and PCI in LCNEC. Future trials are needed to define clinical or pathological markers in LCNEC in order to select patients who may benefit from TRT and PCI.

\section{Conclusions}

Our results suggest that advanced LCNEC should be treated in a similar manner to SCLC in terms of chemotherapy, consolidative TRT and PCI.

\section{Acknowledgements}

The authors thank the research nurses and the patients of Policlinico Umberto I Rome for participating in this study.

\section{References}

1 Oberg K, Castellano D. Current knowledge on diagnosis and staging of neuroendocrine tumors. Cancer Metastasis Rev 2011; 30: Suppl. 1, 3-7.

2 Travis WD. Pathology and diagnosis of neuroendocrine tumors: lung neuroendocrine. Thorac Surg Clin 2014; 24: 257-266.

3 Filosso PL, Ferolla P, Guerrera F, et al. Multidisciplinary management of advanced lung neuroendocrine tumors. J Thorac Dis 2015; 7: S163-S171.

4 Travis WD, Brambilla E, Muller-Hermelink HK, et al. World Health Organization Classification of Tumours. Pathology and Genetics of Tumours of the Lung, Pleura, Thymus and Heart. Lyon, IARC Press, 2004.

5 Travis WD, Bambilla E, Burke AP, et al. WHO Classification of Tumours of the Lung, Pleura, Thymus and Heart. 4th Edn. Geneva, WHO Press, 2015.

6 Rieber J, Schmitt J, Warth A, et al. Outcome and prognostic factors of multimodal therapy for pulmonary large-cell neuroendocrine carcinomas. Eur J Med Res 2015; 20: 64

7 Sun JM, Ahn MJ, Ahn JS, et al. Chemotherapy for pulmonary large cell neuroendocrine carcinoma: similar to that for small cell lung cancer or non-small cell lung cancer? Lung Cancer 2012; 77: 365-370.

8 Jones $\mathrm{MH}$, Virtanen $\mathrm{C}$, Honjoh $\mathrm{D}$, et al. Two prognostically significant subtypes of high-grade lung neuroendocrine tumours independent of small-cell and large-cell neuroendocrine carcinomas identified by gene expression profiles. Lancet 2004; 363: 775-781.

9 Rekhtman N, Pietanza MC, Hellmann MD, et al. Next-generation sequencing of pulmonary large cell neuroendocrine carcinoma reveals small cell carcinoma-like and non-small cell carcinoma-like subsets. Clin Cancer Res 2016; 22: 3618-3629.

10 National Comprehensive Cancer Network. NCCN Clinical Practice Guidelines in Oncology (NCCN Guidelines) Small Cell Lung Cancer. Version 1. 2016. www.nccn.org/professionals/physician_gls/pdf/sclc_blocks.pdf. Date last accessed: September 20, 2016. 
11 Masters GA, Temin S, Azzoli CG, et al. Systemic therapy for stage IV non-small-cell lung cancer: American Society of Clinical Oncology Clinical Practice Guideline update. J Clin Oncol 2015; 33: 3488-3515.

12 Rossi G, Cavazza A, Marchioni A, et al. Role of chemotherapy and the receptor tyrosine kinases KIT, PDGFR $\beta$, PDGFR $\alpha$, and Met in large-cell neuroendocrine carcinoma of the lung. J Clin Oncol 2005; 34: 8775-8785.

13 Niho S, Kenmotsu H, Sekine I, et al. Combination chemotherapy with irinotecan and cisplatin for large-cell neuroendocrine carcinoma of the lung: a multicenter phase II study. J Thorac Oncol 2013; 8: 980-984.

14 Le Treut J, Sault MC, Lena H, et al. Multicentre phase II study of cisplatin-etoposide chemotherapy for advanced large-cell neuroendocrine lung carcinoma: the GFPC 0302 study. Ann Oncol 2013; 24: 1548-1552.

15 Igawa S, Watanabe R, Ito I, et al. Comparison of chemotherapy for unresectable pulmonary high-grade non-small cell neuroendocrine carcinoma and small-cell lung cancer. Lung Cancer 2010; 68: 438-445.

16 Makino T, Mikami T, Hata Y, et al. Comprehensive biomarkers for personalized treatment in pulmonary large cell neuroendocrine carcinoma: a comparative analysis with adenocarcinoma. Ann Thorac Surg 2016; 102: 1694-1701.

17 Jeremic B, Shibamoto Y, Nikolic N, et al. Role of radiation therapy in the combined-modality treatment of patients with extensive diseases small-cell lung cancer: a randomized study. J Clin Oncol 1999; 17: 2092-2099.

18 Yee D, Butts C, Reiman A, et al. Clinical trial of post-chemotherapy consolidation thoracic radiotherapy for extensive-stage small cell lung cancer. Radiother Oncol 2012; 102: 234-238.

19 Slotman BJ, van Tinteren H, Praag JO, et al. Use of thoracic radiotherapy for extensive stage small-cell lung cancer: a phase 3 randomised controlled trial. Lancet 2015; 385: 36-42.

20 Slotman B, Faivre-Finn C, Kramer G, et al. Prophylactic cranial irradiation in extensive small-cell lung cancer. N Engl J Med 2007; 357: 664-672.

21 Seto T, Takahashi T, Yamanaka T, et al. Prophylactic cranial irradiation (PCI) has a detrimental effect on the overall survival (OS) of patients (pts) with extensive disease small cell lung cancer (ED-SCLC): results of a Japanese randomized phase III trial. J Clin Oncol 2014; 32: Suppl. 5, Abstr 7503.

22 Metro G, Ricciuti B, Chiari R, et al. Survival outcomes and incidence of brain recurrence in high-grade neuroendocrine carcinomas of the lung: implications for clinical practice. Lung Cancer 2016; 95: 82-87.

23 Fasano M, Maria Della Corte C, Papaccio F, et al. Pulmonary large-cell neuroendocrine carcinoma: from epidemiology to therapy. J Thorac Oncol 2015; 10: 1133-1141.

24 Eisenhauer EA, Therasse P, Bogaerts J, et al. New response evaluation criteria in solid tumours: revised RECIST guideline (version 1.1). Eur J Cancer 2009; 45: 228-247.

25 Kaplan E, Meier P. Nonparametric estimation from incomplete observations. J Am Stat Assoc 1958; 53: 457-481.

26 Mazières J, Daste G, Molinier L, et al. Large cell neuroendocrine carcinoma of the lung: pathological study and clinical outcome of 18 resected cases. Lung Cancer 2002; 37: 287-292.

27 Iyoda A, Hiroshima K, Moriya Y, et al. Prospective study of adjuvant chemotherapy for pulmonary large cell neuroendocrine carcinoma. Ann Thorac Surg 2006; 82: 1802-1807.

28 Kenmotsu H, Niho S, Ito T, et al. A pilot study of adjuvant chemotherapy with irinotecan and cisplatin for completely resected high-grade pulmonary neuroendocrine carcinoma (large cell neuroendocrine carcinoma and small cell lung cancer). Lung Cancer 2014; 84: 254-258.

29 Yamazaki S, Sekine I, Matsuno Y, et al. Clinical responses of large cell neuroendocrine carcinoma of the lung to cisplatin-based chemotherapy. Lung Cancer 2005; 49: 217-223.

30 Kozuki T, Fujimoto $\mathrm{N}$, Ueoka $\mathrm{H}$, et al. Complexity in the treatment of pulmonary large cell neuroendocrine carcinoma. J Cancer Res Clin Oncol 2005; 131: 147-151.

31 Tokito T, Kenmotsu H, Watanabe R, et al. Comparison of chemotherapeutic efficacy between LCNEC diagnosed using large specimens and possible LCNEC diagnosed using small biopsy specimens. Int J Clin Oncol 2012; 19: 63-67.

32 Shimada Y, Niho S, Ishii G, et al. Clinical features of unresectable high-grade lung neuroendocrine carcinoma diagnosed using biopsy specimens. Lung Cancer 2012; 75: 368-373.

33 Takada M, Fukuoka M, Kawahara M, et al. Phase III study of concurrent versus sequential thoracic radiotherapy in combination with cisplatin and etoposide for limited-stage small-cell lung cancer: results of the Japan Clinical Oncology Group Study 9104. J Clin Oncol 2002; 20: 3054-3060.

34 De Ruysscher D, Pijls-Johannesma M, Bentzen SM, et al. Time between the first day of chemotherapy and the last day of chest radiation is the most important predictor of survival in limited-disease small-cell lung cancer. J Clin Oncol 2006; 24: 1057-1063.

35 Socinski MA, Bogart JA. Limited-stage small-cell lung cancer: the current status of combined-modality therapy. J Clin Oncol 2007; 25: 4137-4145.

36 Palma DA, Warner A, Louie AV, et al. Thoracic radiotherapy for extensive stage small-cell lung cancer: a meta-analysis. Clin Lung Cancer 2015; 17: 239-244.

37 Zhu H, Zhou Z, Wang Y, et al. Thoracic radiation therapy improves the overall survival of patients with extensive-stage small cell lung cancer with distant metastasis. Cancer 2011; 117: 5423-5431.

38 Giuliani ME, Atallah S, Sun A, et al. Clinical outcomes of extensive stage small cell lung carcinoma patients treated with consolidative thoracic radiotherapy. Clin Lung Cancer 2011; 12: 375-379.

39 Luan Z, Wang Z, Huang W, et al. Efficacy of 3D conformal thoracic radiotherapy for extensive-stage small-cell lung cancer: a retrospective study. Exp Ther Med 2015; 10: 671-678.

40 Auperin A, Arriagada R, Pignon JP, et al. Prophylactic cranial irradiation for patients with small-cell lung cancer in complete remission. Prophylactic Cranial Irradiation Overview Collaborative Group. N Engl J Med 1999; 341: 476-484.

41 Le Péchoux C, Sun A, Slotman BJ, et al. Prophylactic cranial irradiation for patients with lung cancer. Lancet Oncol 2016; 17: e277-e293. 